\title{
LA IMPORTANCIA DE LAS PERSONAS EN LA PRESERVACIÓN DE LA NATURALEZA
}

\author{
THE IMPORTANCE OF PEOPLE IN THE PRESERVATION OF NATURE
}

\section{L'IMPORTANCE DES PERSONNES DANS LA PRÉSERVATION DE LA NATURE}

\author{
Mathew G. Linkies \\ University of Kent, UK \\ MG-Linkies@kent.ac.uk
}

(Tipo de artículo: REFLEXIÓN. Recibido el 20/01/2011. Aprobado el 28/03/2011)

\begin{abstract}
RESUMEN
La restauración del capital natural es una de las ideas más radicales que ha surgido en los últimos años, debido a que involucra dos conceptos cuyos exponentes han estado enfrentados por décadas. Por un lado, la necesidad de proteger la biodiversidad y conservar los recursos naturales, tal como lo proponen los conservacionistas y los ambientalistas, y por el otro, la demanda por recursos naturales, según lo proponen los economistas y gran parte de la sociedad. Es necesario trabajar juntos a través de las fronteras disciplinarias tradicionales y las posturas polémicas, con el fin de encontrar soluciones innovadoras que contrarresten la brecha ideológica prevaleciente que separa a la economía y a la ecología. En este trabajo se proponen cinco pilares y tres tareas principales para alcanzar una supervivencia sostenible, incluyendo a la conservación y a la restauración.
\end{abstract}

\section{Palabras clave}

Conservación, restauración, capital natural, capital social, sostenibilidad, ecología.

\section{ABSTRACT}

The restoration of natural capital is one of the most radical ideas that had emerged in recent years, because it involves two concepts whose exponents have been confronted for decades. On the one hand, the need to protect biodiversity and conserve natural resources, as proposed by conservationists and environmentalists, and on the other hand, the demand for natural resources, according to economists and large part of society proposed. It is necessary to work together through traditional disciplinary borders and controversial positions, with the purpose to find innovative solutions to counteract the prevailing ideological gap that separates the economy and ecology. In this work we propose five pillars and three major tasks to reach a sustainable survival, including conservation and restoration.

\section{Keywords}

Conservation, restoration, natural capital, social capital, sustainability, ecology.

\section{RÉSUMÉ}

La restauration de la capitale naturelle est une des idées les plus radicales qui a apparu dans les derniers ans, parce qu'elle implique deux concepts qui ont des représentants qui sont devenus opposants pendant décennies. D'un côté, la nécessité de protéger la biodiversité et garder les ressources naturelles, telle que le proposent les conversationnistes et les environnementalistes, d'une autre côté, la demande de ressources naturelles, comme le proposent les économistes et la plupart de la société. Est nécessaire travailler ensemble à travers des frontières disciplinaires traditionnelle et les postures polémiques, avec l'intention de trouver des solutions innovatrices que compensent la brèche idéologique en vigueur que sépare l'économie et l'écologie. Dans cet article on propose cinq piliers et trois tâches principales pour obtenir une survivance durable, en incluant la conservation et la restauration.

\section{Mots-clés}

Conservation, restauration, capital naturelle, capital sociale, durabilité, écologie. 


\section{INTRODUCCIÓN}

En sólo dos siglos los seres humanos transformaron los ecosistemas terrestres y marinos del planeta y redujeron seriamente las reservas de bienes y servicios esenciales para la vida [1]. Actualmente el planeta está viviendo una situación de rebasamiento ecológico [2]. El propio bienestar de la población, la supervivencia de otras especies y la resistencia de los sistemas mundiales de apoyo a la vida, están todos en riesgo. Por otra parte, el bienestar de la mayoría de las personas -y por lo tanto de la humanidad- realmente ha disminuido en los últimos 35 años [3]. ¿Qué significa esto para la ciencia y la práctica de la conservación? Más que nunca, significa que las personas son parte de la naturaleza y que deben practicar la preservación ecológica -y la restauracióncomo si las personas importaran mucho.

\section{SITUACIÓN ACTUAL}

Actualmente, no sólo biólogos, conservacionistas y ambientalistas, sino también economistas, políticos y líderes empresariales, están cuestionando la eficacia de los paradigmas económicos prevalecientes [4]-[7], y el futuro de lo globalizado: el sistema económico capitalista basado en el consumismo que lo legitima [3]. Ha llegado el momento de unificar esfuerzos para resolver de manera integral los difíciles problemas de las personas, el resto de la naturaleza y los mutuamente independientes sistemas económicos. La Ciencia de la conservación y el uso y manejo racional de las áreas protegidas son parte integral de esta solución, como lo es la restauración ecológica, que es la única esperanza real de incrementar el inventario de los espacios naturales del planeta. La Ecología de la restauración es una de las claves para el futuro de la Ciencia de la conservación [8]-[10], y ambos forman parte de algo más grande, es decir, la Ciencia de la sostenibilidad y la gestión [11].

Los ecologistas y los economistas trabajan en conjunto con ONGs, líderes comunitarios y gobiernos, ayudando a forjar un camino hacia adelante con un nuevo paradigma: ecología como si las personas importaran; economía como si la naturaleza importaba. Cuando se realiza con inclinación práctica tanto como pragmática, y reposa sobre las bases éticas sólidas que sustentan este paradigma, la restauración ecológica -vinculando a la conservación de la naturaleza y la sostenibilidad- ofrece una forma de lograr un diferente y mejor futuro para todos [12] [13]. Obrar para restaurar la naturaleza también es la antítesis y el antídoto a la tesis de la expansión y crecimiento constante del consumismo, que se encuentra embebido en las teorías económicas neoliberales [12]. De hecho, parece que hay una conciencia creciente de que se requiere un cambio profundo, impulsado por la cada vez mayor percepción de los riesgos globales y los retos futuros. Los obstáculos son demasiado grandes y tienen unas bases inadecuadas en la ciencia de los sistemas socio-ecológicos y, sobre todo, en la inercia o la franca oposición en las esferas políticas y económicas.
¿Qué pueden hacer los científicos de la conservación y la restauración? Junto con los esfuerzos diarios de las personas por hacer avanzar las fronteras de la ciencia, es posible ayudar a cruzar los serios abismos informativos, ideológicos y políticos que separan las disciplinas y que son obstáculos en el camino de trabajar juntos por un estilo de vida más sostenible. La conclusión hoy es que el capital natural se ha convertido en el factor limitante para el bienestar humano y la sostenibilidad económica [14] [15]. Anotan estos autores que el capital natural es una noción comprensible por todos y es altamente efectiva para enlazar lo que divide al paradigma subyacente. La restauración ecológica, especialmente si se toma la inversión en restauración del capital natural como el principio rector, puede contribuir tanto a la conservación de la naturaleza como al desarrollo socio-económico sólido y ético, y puede aumentar la oferta de bienes y servicios del ecosistema al tiempo que incrementa el inventario de espacios naturales.

La restauración del capital natural es sin duda una de las ideas más radicales que ha surgido en los últimos años, ya que une dos frentes cuyos exponentes han estado enfrentados durante décadas: la necesidad de proteger la biodiversidad y conservar los recursos naturales, como lo defienden los conservacionistas y ambientalistas, y la demanda por recursos naturales que hacen los economistas y la sociedad en general desde lo local, regional, nacional e internacional. Pero para conseguir que este modelo funcione, es necesario alejarse rápidamente del modelo actual; es necesario trabajar en equipo, o incluso "saltar juntos", a través de las fronteras disciplinarias tradicionales 0 posturas polémicas, con el fin de encontrar soluciones innovadoras que la lucha contra la brecha ideológica que separa la economía y la ecología, así como el conflicto que se percibe entre el desarrollo económico y la conservación de los recursos naturales y del medio ambiente.

\section{PROPUESTA PARA UNA VIDA SOSTENIBLE}

Es necesario demostrar no sólo que la conservación de la naturaleza y la restauración pueden ser rentables, sino que las corporaciones y los gobiernos pueden ayudar rápidamente a promover los objetivos ambientales mediante la restauración simultánea del capital natural y el capital social en las comunidades y los países que sufren de pobreza, desempleo, desesperación y analfabetismo, y la consiguiente pérdida de habilidades, dignidad, autoestima, y carácter social. El capital social se compone de instituciones, relaciones y redes sociales, que comparten creencias y tradiciones culturales y que promueven la confianza mutua. A continuación se presenta la propuesta de los cinco pilares y las tres tareas principales para una vida sostenible, incluida la conservación y restauración.

\section{Los cinco pilares son:}

1. Estructurar e integrar conceptos y modelos ecológicos y económicos 
2. Objetivos basados en valores y ética

3. Estrategias y herramientas efectivas para alcanzar las metas

4. Evaluación rigurosa y multidisciplinar de las actividades y trayectorias de la restauración natural y de capital social

5. Integración socio-económica de proyectos dentro de los contextos culturales, históricos y políticos

Las tres tareas principales son:

1. Desarrollar y probar modelos combinados de conservación, uso sostenible y restauración ecológica que se apliquen y apropien a través de una amplia gama de configuraciones y situaciones biofísicas, históricas, socio-culturales y económicas

2. Comunicar y actuar con eficacia para lograr una vida sostenible mediante una mayor cooperación interdisciplinaria e internacional

3. Restaurar el capital natural y, concomitantemente, el capital social

Sobre este último punto, cabe destacar que las personas que trabajan juntas para restaurar el capital natural también participan activamente en actividades para establecer la confianza mutua y un propósito común, que son las piedras angulares del capital social. Trabajando juntos, será posible encontrar y aplicar soluciones integrales a los problemas acuciantes de las personas y del resto de la Naturaleza.

\section{CONCLUSIONES}

En muchos otros documentos los autores, de diversas partes de la Tierra, muestran que los límites conceptuales y prácticos entre la conservación, la restauración y la gestión de los ecosistemas son cada vez más borrosos, y el rango de paisajes que requiere estudio y la gestión se han vuelto cada vez más complejos [16]. Lo que surge de la lectura de estos trabajos es que, aunque está claro que hay lagunas en la ciencia que necesitan ser atendidas, el principal obstáculo para lograr la restauración a escala local y mundial, es la indiferencias de la sociedad para aceptar la crisis ecológica actual y para hacer frente a lo fundamental del cambio de valoración económica y la inversión. Cambiar el agotamiento de los recursos y la contaminación de una parte del mundo a otra no puede resolver, o incluso ocultar, la erosión a escala mundial del capital social y natural. La era de la doble economía mundial -norte-sur, desarrolladossubdesarrollados- está llegando a su fin. Compartimos un único planeta que necesita atención y reparación.

\section{REFERENCIAS}

[1] Millennium Ecosystem Assessment. "Ecosystems and human well-being: Synthesis". Washington: Island Press. 2005.

[2] M. Wackemagel \& W. E. Rees. "Perceptual and structural barriers to investing in natural capital: Economics from an ecological footprint perspective". Ecological Economics, Vol. 20, No. 1, pp. 3-24. 1997.

[3] J. R. Saul. "The collapse of globalism and the reinvention of the world". Woodstock: The Overlook Press. 2005.

[4] K. Arrow, B. Bolin, R. Costanza, P. Dasgupta, C. Folke, C. S. Holling, B-O. Jansson, S. Levin, K-M. Maler, C. Perrings \& D. Pimentel. "Economic growth, carrying capacity and the environment". Ecological Economics, Vol. 15, No. 2, pp. 91-95. 1995.

[5] J. N. Blignaut. "Towards an economic development ethic". In J. N. Blignaut, \& M. P. de Wit (Eds.), Sustainable options. Cape Town: UCT Press. 2004.

[6] P. Dasgupta. "Human well-being and the natural environment". Oxford: Oxford University Press. 2002.

[7] G. Heal (2000). "Nature and the marketplace". Washington DC: Island Press; CA:Covelo.

[8] A. P. Dobson, A. D. Bradshaw \& A. J. M. Baker. "Hopes for the future: Restoration ecology and conservation biology". Science, Vol. 277, No. 5325, pp. 515-522. 1997.

[9] J. A. MacMahon \& K. D. Holl. "Ecological restoration: A key to conservation biology's future". In M. E. Soule \& G. H. Orians (Eds.). Conservation biology: Research priorities for the next decade. Washington: Island Press. 2001.

[10] T. Young. "Restoration ecology and conservation biology". Biological Conservation, Vol. 92, No. 1, pp. 7383. 2000.

[11] R. W. Kates, W. C. Clark, R. Corell, J. M. Hall, C. C. Jaeger, I. Lowe, J. J. McCarthy, H. J. Schellnhuber, B. Bolin, N. M. Dickson, S. Faucheux, G. C. Gallopin, A. Grübler, B. Huntley, J. Jäger, N. S. Jodha, R. E. Kasperson, A. Mabogunje, P. Matson, H. Mooney, B. Moore III, T. O'Riordan \& U. Svedin. Sustainability science. Science, Vol. 292, pp. 641-642. 2001.

[12] J. N. Blignaut, J. Aronson, S. Archer, A. F. Clewell, P. Woodworth \& N. Desai. "The values and ethics of restoring natural capital". In J. Aronson, S. J. Milton, \& J. N. Blignaut (Eds.) Restoring natural capital: Science, business and practice. Washington: Island Press. 2007.

[13] S. J. Milton, J. Aronson \& J. N. Blignaut. "Restoring natural capital - Shared visions for ecology and economy". Quest (South African Academy of Science), Vol. 2, No. 1, pp. 39-41. 2005.

[14] A. F. Clewell \& J. Aronson. "A global vision for ecological restoration". In press.

[15] J. Aronson, S. J. Milton \& J. N. Blignaut. "Restoring natural capital: Definitions and rationale". In J. Aronson, S. J. Milton, \& J. N. Blignaut (Eds.), Restoring natural capital: Science, business and practice. Washington: Island Press. 2007.

[16] A. Machado. "An index of naturalness". Journal for Nature Conservation, Vol. 12, No. 2, pp. 95-110. 2004. 